\title{
Synthesis of fluorine-18-labelled choline (18F-fluorocholine): towards an early and accurate management of prostate cancer in Malaysia
}

\begin{abstract}
Prostate cancer is ranked fourth among the most prevalent cancers in men in Malaysia. It is anticipated that the number of prostate cancer sufferersôwill increase in future. With the emergence of reputable imaging tracers such as fluorine-18-labelled choline (18Ffluorocholine) and gallium-68 labelled prostate specific membrane antigen (68Ga-PSMA) for the diagnosis of prostate cancer in positron emission tomography / computed tomography (PET/CT) modality, the challenges in the staging and detection accuracy would promise a better management strategy for patients. This article presents the synthesis of $18 \mathrm{~F}-$ fluorocholine and a convenient method for quality control analysis of $18 \mathrm{~F}$-fluorocholine. In addition, the aim of this research work is to assist local Good Manufacturing Practice (GMP) radiopharmaceutical laboratories that routinely produce $18 \mathrm{~F}$-fluorodeoxyglucose (18F-FDG), to start producing $18 \mathrm{~F}$-fluorocholine as a tracer for prostate cancer imaging.
\end{abstract}

Keyword: 18F-fluorocholine; GE TracerLab MXFDG; Positron emission tomography/computed tomography; Quality control analysis; Radiochemical yield 